\title{
Fetal-growth-restricted preterm infants display compromised autonomic cardiovascular control on the first postnatal day but not during infancy
}

\author{
Emily Cohen ${ }^{1}$, Flora Y. Wong ${ }^{1}$, Euan M. Wallace ${ }^{2}$, Joanne C. Mockler ${ }^{3}$, Alexsandria Odoi ${ }^{1}$, Samantha Hollis ${ }^{1}$, \\ Rosemary S.C. Horne ${ }^{1}$ and Stephanie R. Yiallourou ${ }^{1}$
}

BACKGROUND: Fetal growth restriction (FGR) is associated with increased perinatal mortality and long-term cardiovascular and neurodevelopmental sequelae. We hypothesized that FGR impacts on the development of autonomic heart rate and blood pressure control, contributing to unfavorable short- and long-term outcomes following FGR.

METHODS: We studied 25 preterm FGR and 22 preterm and 19 term appropriate for gestational age (AGA) infants. Preterm neonates were studied on postnatal day 1 , and all infants were studied at 1 and 6 months post-term age. To investigate autonomic cardiovascular control, we examined heart rate variability (HRV) and baroreflex sensitivity using spectral power and transfer-function analyses.

RESULTS: Preterm FGR neonates exhibited higher heart rates and reduced HRV compared with preterm AGA controls on postnatal day 1. No significant differences were found between the three groups at 1 or 6 months post-term age.

CONCLUSION: Preterm FGR neonates display compromised HRV on postnatal day 1, which may suggest increased vulnerability to circulatory instability. This may predispose these neonates to systemic and cerebral hypoperfusion and increase the risk of long-term neurodevelopmental sequelae. Differences were no longer found at 1 and 6 months post-term age, suggesting that the maturation of autonomic cardiovascular control may be preserved following FGR.

$\mathbf{F}$ etal growth restriction (FGR) is associated with an increased risk of perinatal and neonatal morbidity and mortality (1). It is also believed to cause adverse long-term neurodevelopmental (2) and cardiovascular (3) sequelae. However, the mechanisms underpinning the increased risk for these adverse outcomes remain unclear. One explanation for the long-term pathophysiology is abnormal development of the autonomic nervous system. Autonomic function can be assessed noninvasively by means of heart rate variability (HRV) and baroreflex sensitivity (BRS) analysis (4). Using these techniques, altered autonomic cardiovascular control in early life has been related to compromised neurodevelopmental outcome in childhood $(5,6)$. Moreover, studies in adults have recognized relative sympathetic hyperactivity as the main pathophysiologic mechanism causing hypertension (7). The development of autonomic cardiovascular control begins during fetal life and continues well into infancy $(8,9)$. Several studies have demonstrated that autonomic cardiovascular control is compromised in FGR fetuses (10-13). However, studies investigating postnatal maturation of autonomic cardiovascular control following FGR are scarce (14). There have also been no longitudinal studies assessing the maturation of autonomic cardiovascular control in prematurely born FGR infants. It is important to take prematurity into account in any assessment of possible impact of FGR, because the majority of growth-restricted fetuses-especially those severely growth-restricted-are born preterm, and prematurity in itself has been shown to impair autonomic function (9).

In this study, we aimed to longitudinally assess the maturation of autonomic cardiovascular control in preterm FGR infants by means of HRV and BRS analysis. We hypothesized that preterm FGR infants would display altered maturation of autonomic cardiovascular control with relative sympathetic hyperactivity compared with appropriately grown (appropriate for gestational age, AGA) peers.

\section{RESULTS}

Table 1 shows the demographic characteristics of the overall study population and subject information at each study. Preterm FGR infants had a similar gestational age at birth compared with preterm AGA infants, but as expected gestational age was lower compared with term AGA infants $(P<0.001)$. Preterm FGR infants were born with a significantly lower birth weight and birth weight $z$-score than preterm AGA and term AGA infants $(P<0.001$ for both).

\footnotetext{
${ }^{1}$ The Ritchie Centre, Hudson Institute of Medical Research and Department of Paediatrics Monash University, Melbourne, Victoria, Australia; ${ }^{2}$ The Ritchie Centre, Department of Obstetrics and Gynaecology, Monash University, Melbourne, Victoria, Australia; ${ }^{3}$ Department of Obstetrics and Gynaecology, Monash University and Monash Women's, Monash Health, Melbourne, Victoria, Australia. Correspondence: Stephanie R. Yiallourou (stephanie.yiallourou@hudson.org.au) 


\section{Autonomic control following FGR Articles}

Table 1. Demographics

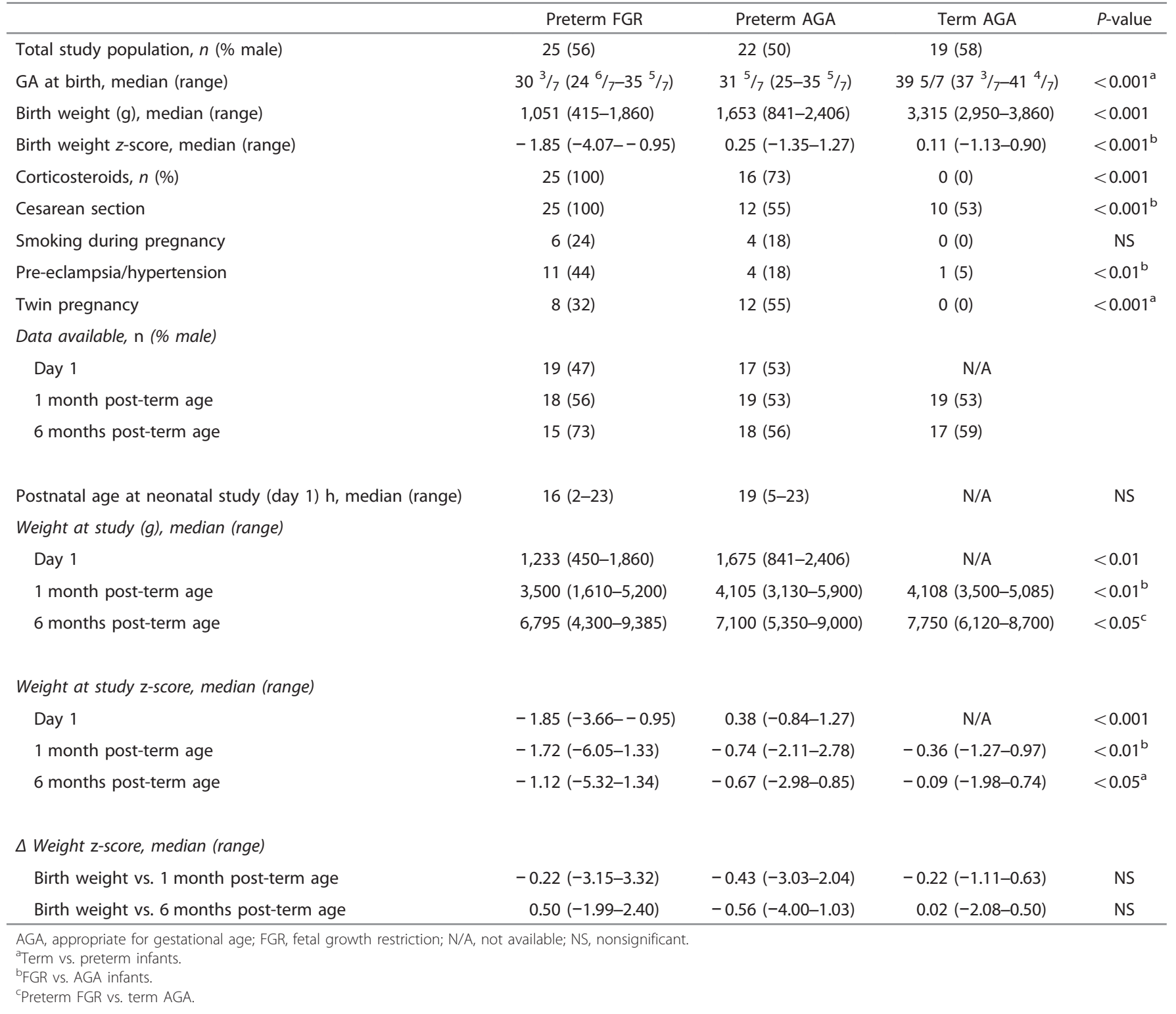

Postnatal growth rate (expressed as the difference between birth weight $z$-score and weight $z$-score at 1 and 6 months post-term age, respectively) was not different between the groups. Table 1 also shows availability of data at each study. Three preterm FGR infants passed away before study completion (age at time of death and cause of death were as follows: infant 1, 3 weeks post-term age, intestinal malrotation, and volvulus; infant 2, 6 weeks post-term age, multisystem failure; and infant 3, 4-month post-term age, severe chronic lung disease). Other missing data were attributable to loss to follow-up, infants unable to attend studies because of illness, and the difficulty of conducting these studies of spontaneous sleep in infants.

\section{Heart Rate and Blood Pressure}

Heart rate and blood pressure data at each study are presented in Table 2. Preterm FGR infants had higher heart rates than preterm AGA peers in active sleep (AS) (140 vs. $129 \mathrm{bpm}$, $P<0.05)$ and quiet sleep (QS) (142 vs. $127 \mathrm{bpm}, P=0.051)$ on day 1 . Heart rate and blood pressure were not different between groups at 1 or 6 months post-term age.

\section{Heart Rate Variability}

All HRV indices were significantly positively correlated with $\mathrm{R}-\mathrm{R}$ interval at each age in both sleep states $(P<0.001$ for most). On day 1 , gestational age showed significant positive correlations with all HRV indices in AS 


\section{Articles | Cohen et al.}

Table 2. Heart rate and blood pressure

\begin{tabular}{|c|c|c|c|c|}
\hline & Preterm FGR & Preterm AGA & Term AGA & $P$-value \\
\hline \multicolumn{5}{|l|}{ Heart rate AS } \\
\hline Day 1 & $140(117-171)$ & 129 (116-153) & N/A & 0.014 \\
\hline 1 month & $136(115-158)$ & 135 (123-152) & $136(110-153)$ & NS \\
\hline 6 months QS & 116 (109-141) & $121(98-137)$ & $123(108-131)$ & NS \\
\hline
\end{tabular}

\begin{tabular}{|c|c|c|c|c|}
\hline Day 1 & $142(112-166)$ & 127 (116-152) & $\mathrm{N} / \mathrm{A}$ & 0.051 \\
\hline 1 month & 133 (114-149) & 130 (119-149) & 133 (107-149) & NS \\
\hline 6 months & $111(100-136)$ & $114(87-126)$ & $112(98-138)$ & NS \\
\hline
\end{tabular}

SBP AS

$\begin{array}{lllll}1 \text { month } & 87(60-119) & 88(69-114) & 93(83-117) & \text { NS } \\ 6 \text { months } & 85(71-102) & 85(69-100) & 91(81-115) & \text { NS }\end{array}$

SBP QS

$\begin{array}{lllll}1 \text { month } & 74(54-97) & 75(56-104) & 77(60-114) & \text { NS } \\ 6 \text { months } & 77(63-93) & 78(64-100) & 85(73-94) & \text { NS }\end{array}$

DBP AS

$\begin{array}{lllll}1 \text { month } & 55(32-84) & 62(46-85) & 67(56-82) & \text { NS } \\ 6 \text { months } & 60(43-80) & 59(44-83) & 66(54-84) & \text { NS }\end{array}$

$D B P Q S$

$\begin{array}{lllll}1 \text { month } & 49(35-64) & 53(35-82) & 56(41-83) & \text { NS } \\ 6 \text { months } & 55(37-72) & 55(42-77) & 60(48-73) & \text { NS }\end{array}$

MABP AS

$\begin{array}{lllll}1 \text { month } & 69(41-96) & 72(55-95) & 76(66-93) & \text { NS } \\ 6 \text { months } & 69(52-87) & 67(53-89) & 75(64-94) & \text { NS }\end{array}$

MABP QS

$\begin{array}{lllll}1 \text { month } & 56(42-73) & 59(42-90) & 63(49-93) & \text { NS } \\ 6 \text { months } & 64(46-79) & 64(50-85) & 69(56-80) & \text { NS }\end{array}$

AGA, appropriate for gestational age; AS, active sleep; DBP, diastolic blood pressure; FGR, fetal growth restriction; MABP, mean arterial blood pressure; NS, nonsignificant; QS, quiet sleep; SBP, systolic blood pressure.

(low-frequency (LF)/high-frequency (HF) $r=0.49, P<0.01$, LF $r=0.66, P<0.001$, HF $r=0.33, P<0.05$, total power (TP) $r=0.63, P<0.001)$ and all but $\mathrm{HF}$ in QS (LF/HF $r=0.45$, $P<0.05$, LF $r=0.6, P<0.001$, TP $r=0.53, P<0.01)$. Birth weight $z$-score also showed significant positive correlations with TP in QS $(r=0.40, P<0.05)$ and AS $(r=0.35, P<0.05)$ on day 1. A relationship between birth weight $z$-score and LF was also observed in both sleep states on day 1 ; however, this just failed to reach statistical significance (QS $r=0.36$, $P=0.052$, AS $r=0.33, P=0.051)$. HRV indices were not correlated with postnatal growth rate ( $\Delta$ weight $z$-score). Figure 1 shows the effect of size at birth and postnatal age on HRV. All HRV parameters changed significantly with advancing postnatal age $(P<0.001$ for all). The $\mathrm{LF} / \mathrm{HF}$ ratio decreased, whereas LF, HF, and TP showed an increase with advancing postnatal age. Postnatal age and group demonstrated a significant interaction for LF and TP in both sleep states $(P<0.05$ for both). Further testing revealed that differences were only present on day 1, with preterm FGR neonates demonstrating reduced LF and TP than their AGA peers in both sleep states (LF QS $P$ 0.015, F 6.7, $r$ 0.439, LF AS $P 0.016, \mathrm{~F} 6.4, r 0.397$, TP QS $P 0.010$, F 7.6, $r 0.462$, TP AS $P$ $0.010, \mathrm{~F} 7.4, r$ 0.422). When the model was corrected for $\mathrm{R}-\mathrm{R}$ interval, however, these group differences were no longer detectable.

\section{Baroreflex Sensitivity}

Figure 2 shows BRS data at 1 and 6 months of age in AS and QS. BRS showed a significant increase with advancing postnatal age in both AS and QS ( $P<0.001$ for both). BRS maturation was not different between the groups.

\section{DISCUSSION}

To our knowledge, this is the first study to report longitudinal autonomic cardiovascular data from birth until 6 months post-term age in preterm FGR infants. We have shown that preterm FGR neonates have compromised HRV compared with preterm AGA peers on the first day of life. However, at 1 and 6 months post-term age, we observed no differences in heart rate, blood pressure, HRV, or BRS between preterm FGR infants and preterm and term AGA peers.

Several studies have demonstrated reduced HRV in FGR fetuses compared with AGA peers, supportive of the concept that these fetuses are compromised by the unfavorable intrauterine environment (10-12). The results of our study confirm the findings of Spassov et al. (15), indicating that infants born growth-restricted may continue to display lowered HRV than AGA peers throughout the first days of life. The highly significant positive correlations between gestational age and HRV indices on postnatal day 1 confirm gradual maturation of autonomic cardiovascular control throughout pregnancy (16). These combined findings suggest that HRV is influenced by both prematurity and FGR, separately. Compromised HRV may reflect impaired autonomic cardiovascular control, predisposing neonates to circulatory instability. A recent study by our group demonstrated reduced HRV in preterm neonates requiring inotropic support for hemodynamic instability in the first 3 days of life. It was suggested that impaired autonomic cardiovascular control could underpin the hemodynamic failure that manifests clinically as hypotension (17). The results of the current study would thus imply that preterm FGR neonates may be more vulnerable to hemodynamic instability during the immediate postnatal period. This may predispose these infants to systemic and cerebral hypoperfusion and increase the risk of long-term neurodevelopmental sequelae. Thus, it 

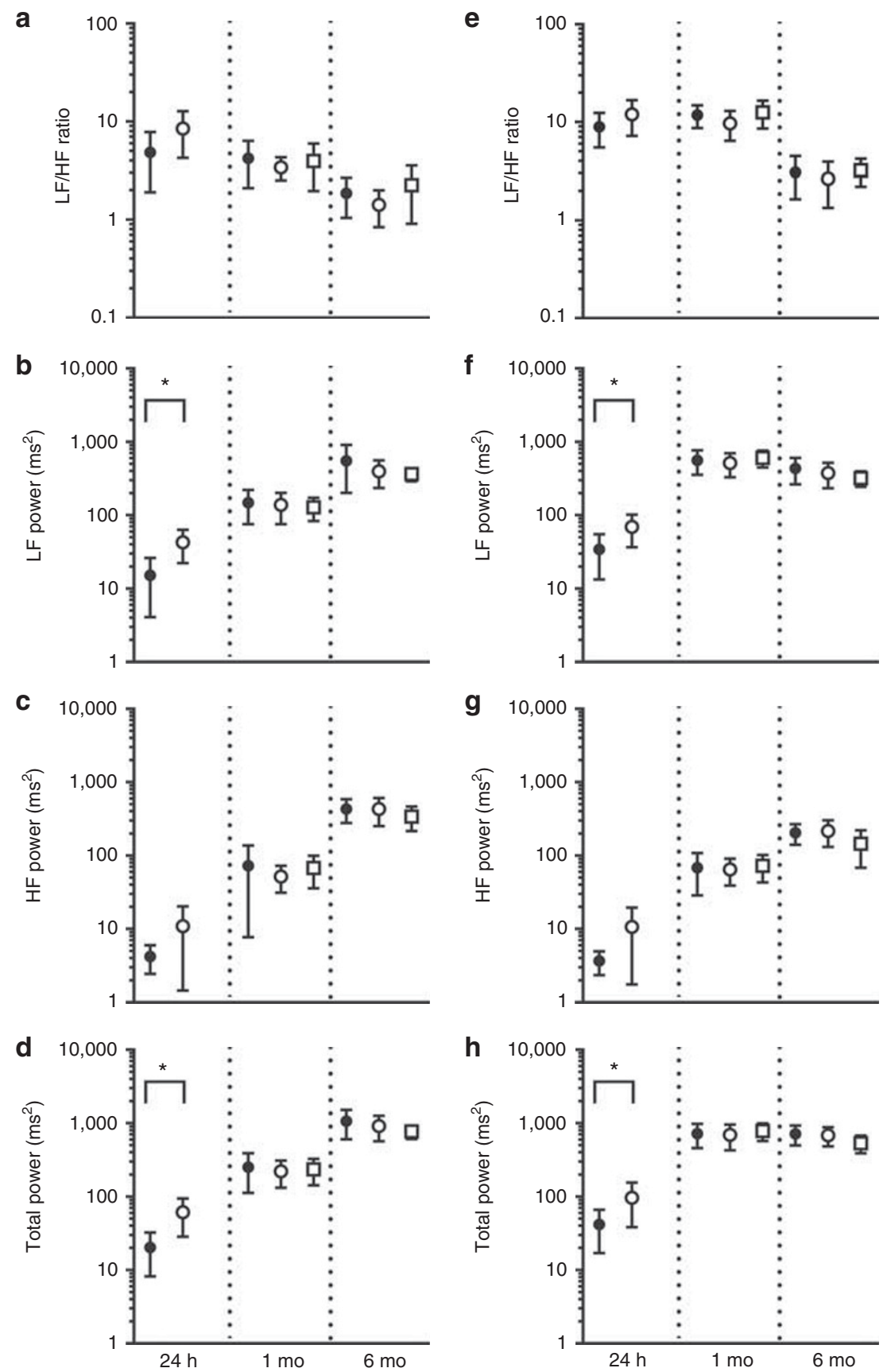

Figure 1. Effect of size at birth and maturation on low-frequency (LF)/high-frequency (HF) ratio, LF power, HF power, and total power (TP) during quiet sleep (QS) (a-d) and active sleep (AS) (e-h). The preterm fetal growth restriction (FGR) group is represented by the closed circles, the preterm appropriate for gestational age (AGA) group by the open circles, and the term AGA group by the open squares. $N$-numbers for the preterm FGR, preterm AGA, and term AGA group, respectively, in QS on day 1: 13, 17, N/A, at 1 month: 18, 18, 18, and at 6 months: 15, 18, 17, and in AS on day 1: $19,17, \mathrm{~N} / \mathrm{A}$, at 1 month: $18,15,18$, and at 6 months: $13,17,14 .{ }^{*} P<0.0167$.

can be postulated that long-term neurodevelopmental sequelae in preterm FGR infants may be related to the cerebral hemodynamic consequences of compromised heart rate and blood pressure control, especially in the early postnatal period. The cerebral vasculature aims to maintain fairly constant cerebral blood flow despite fluctuations in systemic blood pressure (cerebrovascular autoregulation) (18). It is thought that cerebrovascular autoregulation is impaired in preterm neonates, which predisposes the brain to fluctuations in cerebral perfusion, leading to brain injury (18). Animal and human studies have indicated that FGR alters structural and functional properties of the cerebral vasculature, which may further compromise cerebrovascular autoregulation and increase vulnerability of the brain to hemodynamic instability 


\section{Articles | Cohen et al.}
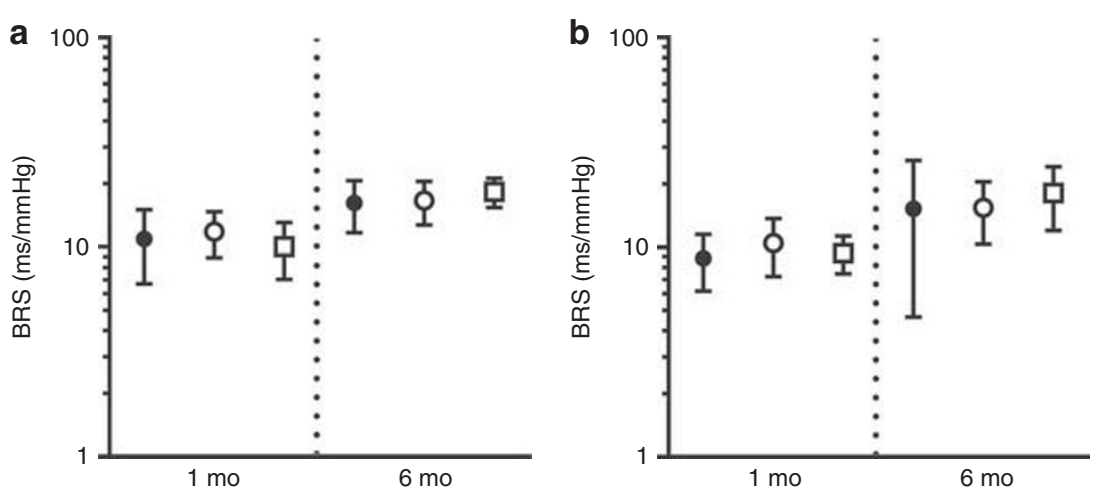

Figure 2. Baroreflex sensitivity (BRS) during quiet sleep (QS) (a) and active sleep (AS) (b) at 1 and 6 months post-term age. The preterm fetal growth restriction (FGR) group is represented by the closed circles, the preterm appropriate for gestational age (AGA) group by the open circles, and the term AGA group by the open squares. $N$-numbers for the preterm FGR, preterm AGA, and term AGA group, respectively, in QS at 1 month:18, 17, 18, and at 6 months: 13,16,17, and in AS at 1 month: 13, 12, 13, and at 6 months: 5, 10, 10.

(19). In fact, hemodynamic instability due to a patent ductus arteriosus has been shown to cause a greater fall in cerebral oxygenation in preterm FGR neonates than in preterm AGA peers (20). It thus appears that FGR brains may be more vulnerable to systemic and cerebral hypoperfusion, potentially increasing the risk of long-term neurodevelopment sequelae.

It could also be postulated, however, that compromised $\mathrm{HRV}$ is a consequence of altered neurodevelopment due to FGR (21). HRV is believed to be an effective tool to study not only cardiovascular function but also general maturation of the central nervous system $(5,6)$. Several retrospective studies have revealed that children with compromised neurodevelopmental outcomes displayed reduced HRV during their neonatal period $(5,6,22,23)$. Compromised HRV in our FGR neonates may thus also be reflective of suboptimal brain development in utero (24).

Previous studies have indicated a close inverse relationship between heart rate and HRV, and it has thus been suggested that the analysis needs to be adjusted for R-R interval (25). FGR neonates demonstrated higher heart rates than their AGA peers on postnatal day 1 . When $R-R$ interval was included in the model, we could no longer detect differences in HRV between preterm FGR and AGA neonates on the first postnatal day. This finding may underline the importance of $\mathrm{R}-\mathrm{R}$ correction in HRV analysis. However, increased heart rate and reduced HRV are both thought to be the result of an imbalance in autonomic cardiovascular control. In fact, in a study in infants with congenital heart disease, increased heart rate and reduced heart rate variability were reported alongside elevated levels of norepinephrine, indicating autonomic imbalance (26). Moreover, a study in adults found significant and independent correlations between increased heart rate and reduced HRV and cardiovascular risk factors (27). The increased heart rate and reduced HRV as observed in our FGR group may thus both be due to an imbalance of autonomic cardiovascular control in the immediate postnatal period. A limited number of studies have indicated relative sympathetic hyperactivity in FGR neonates and infants
(28-30). Although our FGR neonates demonstrated increased heart rates on postnatal day 1 , which may indicate increased sympathetic activity, the HRV parameters did not provide evidence for sympathetic hyperactivity at any postnatal age. LF, representing both sympathetic and parasympathetic activity, was reduced on postnatal day 1 . Moreover, the LF/HF ratio providing information regarding the sympathovagal balance was not different from AGA controls at any age.

Our study is the first to longitudinally investigate maturation of autonomic cardiovascular control in preterm FGR infants until 6 months post-term age. To investigate the effect of the unfavorable intrauterine environment, preterm infants were studied within the immediate postnatal period. Infants were subsequently studied at 1 and 6 months post-term age to investigate maturation of autonomic cardiovascular control. In contrast to our hypothesis, at follow-up no differences in HRV or BRS were found between the groups, indicating that early alterations in autonomic cardiovascular control had been resolved. These findings may suggest that alterations in HRV in preterm FGR neonates predominantly reflect their compromised intrauterine condition, yet maturation of autonomic cardiovascular control throughout infancy is preserved. Nevertheless, studies in FGR children and young adults have demonstrated compromised HRV, BRS, and blood pressure rhythmicity compared with AGA controls (31-33). It can therefore be speculated that FGR infants may initially be capable of restoring autonomic function, but during further development and under the influence of other risk factors-such as excessive catch-up growth-cardiovascular control may again become compromised and gradual progression into chronic cardiovascular disease may occur. Indeed, in the study by Aziz et al. (31) FGR children with the lowest birth weight yet greatest weight gain during childhood showed reduced HRV compared with both FGR and AGA children with less marked weight gain. Dalla Pozza et al. (32) demonstrated that within their FGR population those with increased blood pressure and reduced BRS were also heavier and had a greater body mass index. Furthermore, in the study 
by Wolfenstetter et al. (33), blood pressure rhythmicity was independently influenced by catch-up growth in FGR children. The FGR infants in our study did not show evidence of (excessive) catch-up growth, which may in part explain why maturation of autonomic cardiovascular control was unaffected. It could thus be postulated that infancy may create a window of opportunity for interventions, such as monitoring weight gain to avoid excessive catch-up growth.

A few cases in the preterm FGR group $(n=4)$ had a birth weight above the 10th percentile for their gestational age (birth weight $z$-score $>-1.28$ ). Some may argue that these infants were incorrectly classified as having FGR. However, controversy exists regarding the most appropriate definition of FGR (14). The traditional definition of a birth weight $<10$ th percentile for gestational age on the normative population growth curve can lead to the misdiagnosis of constitutionally small fetuses and may fail to identify larger fetuses who were in fact growth-restricted. It thus appears important to not only consider birth weight but to take other markers of fetal well-being into account. A recent study by Khalil et al. (34) has provided evidence that prenatal Doppler measurements can correctly identify fetuses with impaired growth velocity and pregnancies at risk of adverse outcome, even when fetal size is considered AGA. Our definition of FGR was based on abnormal fetal growth (estimated fetal weight below the 10th percentile and/or slowing of fetal growth velocity) in combination with evidence of placental insufficiency as demonstrated by abnormal multivessel integrated Doppler analysis. Moreover, all of the fetuses in our preterm FGR group were delivered prematurely because of suspected fetal compromise. We thus believe that all infants in the preterm FGR group represent true cases of FGR.

We acknowledge the limitations of this study. First, it is important to note that most studies in FGR infants used headup tilting to investigate autonomic cardiovascular control (28-30). Subtle alterations in autonomic cardiovascular control may only become apparent when the infant is exposed to a stressor such as a head-up tilt (29). In our study, the infants were investigated in a resting state only, which may have limited our ability to detect more subtle changes in autonomic cardiovascular control and sympathovagal balance. However, we have previously demonstrated good levels of agreement between BRS analysis of spontaneous blood pressure fluctuations and BRS analysis following a head-up tilt (35). Second, we were unable to obtain data for each infant at each age. To overcome this issue, we used a mixed model design for our statistical analysis. This method allows for missing at random data and we are thus confident of the robustness of our findings. Last, we cannot exclude a confounding effect of prenatal corticosteroid administration, maternal (perinatal) drug administration, and mode of delivery on neonatal HRV. Prenatal corticosteroid administration was significantly more common in preterm FGR neonates than in their preterm AGA peers. Although the effect of prenatal corticosteroid administration on postnatal HRV remains largely unknown, studies have demonstrated a biphasic response of fetal HRV to corticosteroid administration (36): after an initial increase in fetal HRV, a decrease in HRV is observed before return to baseline $\sim 4$ days after administration. It could be speculated that-depending on the timing of delivery-prenatal corticosteroid administration may affect neonatal HRV. Maternal (perinatal) drug administration has also been shown to affect fetal HRV parameters (37). However, information on maternal (perinatal) drug administration was inconsistently recorded in our research database. Mode of delivery was significantly different between the groups. However, the effect of mode of delivery on neonatal HRV remains largely unknown. To our knowledge, only one study has investigated this matter. In the study by Sheen et al. (38), a reduction in HF and TP was found in neonates delivered via cesarean section compared with neonates delivered via normal vaginal birth. However, the cesarean section group was heterogeneous and contained both elective cesarean sections and cesarean sections performed for nonreassuring fetal status. Within the cesarean section group, neonates delivered for nonreassuring fetal status demonstrated reduced HF and TP compared with neonates delivered via elective cesarean section. The observed differences in neonatal HRV following cesarean section vs. vaginal delivery may thus (in part) be explained by cases of fetal compromise in the cesarean section group. Moreover, all neonates studied were born at term. To our knowledge, no studies have investigated the effect of mode of delivery on neonatal HRV in preterm infants. A larger study would be necessary to account for these potential confounding variables.

\section{CONCLUSIONS}

Preterm FGR neonates display compromised HRV on the first postnatal day, which suggests increased vulnerability to circulatory instability in the immediate postnatal period. This may predispose these infants to systemic and cerebral hypoperfusion and increase the risk of long-term neurodevelopmental sequelae. Differences were no longer found at 1 and 6 months post-term age, suggesting that maturation of autonomic cardiovascular control may be preserved following FGR.

\section{METHODS}

Between June 2013 and December 2015, 25 preterm FGR infants, 22 preterm AGA infants, and 19 term AGA infants were recruited from Monash Medical Centre, Melbourne, Australia. Preterm FGR infants were identified antenatally based on an estimated fetal weight below the 10th percentile for gestational age and/or slowing fetal growth velocity (39) in combination with abnormal multivessel integrated Doppler analysis (uterine artery (40), umbilical artery (41), and middle cerebral artery (42)). All preterm FGR infants were delivered via cesarean section on the basis of suspected fetal compromise. Preterm AGA infants were identified based on normal fetal growth with normal uteroplacental Doppler studies (if performed). Preterm AGA infants were born following spontaneous preterm delivery or were delivered on the basis of suspected fetal compromise of their FGR twin. For logistic reasons, only preterm FGR and preterm AGA infants with a gestational age at birth below 36 weeks who were admitted to Monash Newborn Services were eligible to participate. Term AGA infants were recruited from our postnatal ward or via 
word of mouth. Term AGA infants were born from uncomplicated pregnancies beyond 37 weeks gestational age and from mothers without significant medical history. Exclusion criteria were known congenital or chromosomal abnormalities. Eligible cases were approached to participate in the study. The main reason for families to decline study participation was inability to attend Monash Medical Centre for the follow-up studies (described below). This study was approved by Monash Health and Monash University Human Research Ethics Committees and parental written informed consent was obtained for all participants.

\section{Data Acquisition}

It is known that autonomic cardiovascular control is influenced by the behavioral state of the infant (8). To account for this, data were collected during a daytime sleep study. To investigate the effect of the unfavorable intrauterine environment, preterm infants were studied on postnatal day 1 . All infants were studied at 1 and 6 months postterm age to investigate maturation of autonomic cardiovascular control. These ages were specifically chosen as heart rate variability has been shown to undergo most rapid maturation between these post-term ages (43).

\section{Data Acquisition Neonatal Studies}

Preterm neonates underwent a 2-h sleep study in the neonatal intensive care unit on postnatal day 1. Continuous electrocardiograms (ECG) as part of routine clinical care were collected and digitalized for analysis (PowerLab 16SP, ADInstruments, New South Wales, Australia; LabChart V6, ADInstruments, Sydney, New South Wales, Australia). A sampling rate at or above $512 \mathrm{~Hz}$ was used. Neonates were studied in the supine position and sleep state was scored at cot side as AS or QS based on behavioral criteria (44). Owing to the logistic reasons, no data were acquired in term AGA neonates on postnatal day 1 .

\section{Data Acquisition Infant Studies}

All groups underwent daytime polysomnography in our sleep laboratory at 1 and 6 months post-term age. ECG data were recorded with a sampling rate of $512 \mathrm{~Hz}$. In addition to ECG, electrophysiological signals required for sleep state scoring were recorded and included electroencephalogram, electro-oculogram, submental electromyogram, and abdominal and thoracic respiratory effort (zRIP Durabelt Pediatric, Pro-Tech, Mukilteo, WA). Arterial oxygen saturation was recorded via pulse oximetry (Masimo Australia Pty Ltd, Frenchs Forest, NSW, Australia). In addition to standard polysomnographic recordings, blood pressure was measured noninvasively and continuously using a photoplethysmographic cuff placed around the infant's wrist (Finometer, FMS, Finapres Medical Systems, the Netherlands). To avoid venous pooling in the infant's hand, blood pressure was recorded in 2 min epochs, followed by a 2 min rest period (45). All infants were studied in the supine position. PSG data were recorded using a commercially available PSG system (E-Series; Compumedics, Melbourne, Victoria, Australia). Data were transferred via EDF format to LabChart V6 (ADInstruments, Sydney, New South Wales, Australia) for analysis. Sleep state was scored off-line using standard infant criteria (46).

\section{Data Analysis}

Data analysis was performed off-line (LabChart V6, ADInstruments, Sydney, New South Wales, Australia). To investigate autonomic cardiovascular control, we performed HRV and BRS analysis (4). ECG and blood pressure data interrupted by movement, handling, respiratory events, and arousals were excluded from analysis. Artifact-free ECG data were divided into sleep-state-specific epochs of 1-2 min duration for power spectral analysis of HRV (45). Power spectral analysis allows decomposition of heart rate changes in LF and HF fluctuations. LF changes in heart rate are attributed to baroreflex-mediated influences and reflect both sympathetic and parasympathetic activity, whereas HF changes in heart rate are associated with respiratory activity and represent the fast-acting parasympathetic branch only. The ratio between LF and HF HRV (LF/HF) indicates sympathovagal balance (8). Data were visually inspected and outliers in $\mathrm{R}-\mathrm{R}$ intervals, as defined as an $\mathrm{R}-\mathrm{R}$ interval exceeding $20 \%$ of the average R-R intervals of the epoch (4), were excluded from analysis. Fast Fourier transform (MATLAB R2012b, Mathworks: Natick, MA) with set frequency bands was used to compute the spectral power for the R-R series (45). The LF band was set at $0.04-0.15 \mathrm{~Hz}$, and the HF band was set at an age-dependent threshold based on observed infant respiratory rates during the studies (postnatal day 1: $0.4-1.5 \mathrm{~Hz}, 1$ month post-term age: $0.33-$ $1.3 \mathrm{~Hz}, 6$ months post-term age: $0.25-1.0 \mathrm{~Hz}$ ). The total frequency (total power; TP) band of interest was $0.04-1.5 \mathrm{~Hz}$ (47). Data were downsampled to $200 \mathrm{~Hz}$ during analysis. Average R-R interval as generated by HRV analysis was used to calculate average heart rate. Artifact-free 1-2 min epochs with simultaneous blood pressure and ECG recordings were also analyzed to assess baroreflex function. The baroreflex is responsible for short-term blood pressure control. The reflex aims to buffer sudden changes in blood pressure by modifying heart rate and vascular resistance (48). Baroreflex function can be assessed by BRS analysis, which examines the heart rate change in response to a unit change in blood pressure (48). Cross-spectral (transfer function) analysis between spontaneous LF fluctuations of $\mathrm{R}-\mathrm{R}$ and systolic blood pressure was performed to estimate BRS, as described previously (49). In short, transfer-function analysis was used to determine transfer gain, phase, and coherence between systolic blood pressure and R-R in the LF band $(0.04-0.15 \mathrm{~Hz})$. Transfer gain reflects the degree to which changes within systolic blood pressure become manifest in the R-R signal ( $\mathrm{ms} / \mathrm{mmHg}$ ). The value of transfer gain at the frequency of maximum coherence within the LF band was chosen to estimate BRS. BRS values with corresponding negative phase delay were excluded, as this implies that the change in $\mathrm{R}-\mathrm{R}$ precedes the change in systolic blood pressure and therefore is not due to baroreflex activity. The first $10 \mathrm{~s}$ following internal calibration of each blood pressure measurement were used to determine systolic, diastolic, and mean arterial blood pressure. Data were averaged for each sleep state at each age for each infant before statistical analysis.

\section{Statistical Analysis}

Data were statistically analyzed using IBM SPSS Statistics for Windows, Version 22.0 (IBM, Released 2013, Armonk, NY). Data were checked for normality and outliers by visual assessment of distribution graphs. Chi-square, Fisher exact, or Kruskal-Wallis with step-wise step-down procedure were used as appropriate to analyze demographics and absolute heart rate and blood pressure data. Spearman's correlation was calculated between demographics and HRV and BRS data for each study. HRV and BRS data were logtransformed and analyzed using a mixed model approach to explore the effect of age, group, and their interaction. HRV data were explored with and without $\mathrm{R}-\mathrm{R}$ interval as a covariate in the model (25). Statistically significant results were followed up with single ANOVAs for which the a-level was adjusted for multiple testing using the Bonferroni correction $(0.05 / 3=0.0167)$. All data were analyzed separately for quiet and active sleep to avoid the confounding effect of sleep state (8). Clinical data are presented as $n$ (\%) or median (range). For HRV and BRS, raw data are represented as mean with $95 \%$ confidence interval. The HRV LF, $\mathrm{HF}$, and TP indices are presented as absolute power, in $\mathrm{ms}^{2}$, and BRS in $\mathrm{ms} / \mathrm{mm} \mathrm{Hg}$.

\section{ACKNOWLEDGMENTS}

This project was supported by the National Health and Medical Research Council of Australia Project APP1045559 and Red Nose, Australia. We thank all parents and infants who participated in the study. We also thank the staff at the Fetal Monitoring Unit of Monash Health for their help with recruitment and the staff of Monash Newborn and the Melbourne Children's Sleep Centre where these studies were carried out. 


\section{Autonomic control following FGR $\mid$ Articles}

\section{STATEMENT OF FINANCIAL SUPPORT}

This research was funded by the National Health and Medical Research Council of Australia (APP1045559 to SRY, EMW and RSCH). Funding was provided by the Victorian government's operational infrastructure support program. A scholarship was provided to EC by Red Nose (formerly SIDS and Kids). FW is supported by a National Health and Medical Research Council of Australia Career Development Fellowship.

Disclosure: The authors declare no conflicts of interest.

\section{REFERENCES}

1. Garite TJ, Clark R, Thorp JA. Intrauterine growth restriction increases morbidity and mortality among premature neonates. Am J Obstet Gynecol 2004;191:481-7.

2. Baschat AA. Neurodevelopment following fetal growth restriction and its relationship with antepartum parameters of placental dysfunction. Ultrasound Obstet Gynecol 2011;37:501-14.

3. Barker DJ, Winter PD, Osmond C, Margetts B, Simmonds SJ. Weight in infancy and death from ischaemic heart disease. Lancet 1989;2:577-80.

4. Taylor JA, Lipsitz LA. Heart rate variability. Standards of measurement, physiological interpretation, and clinical use. Task force of the european society of cardiology and the north american society of pacing and electrophysiology. Eur Heart J 1996;17:354-81.

5. Dimitrijevic L, Bjelakovic B, Colovic $\mathrm{H}$, et al. Assessment of general movements and heart rate variability in prediction of neurodevelopmental outcome in preterm infants. Early Hum Dev 2016;99:7-12.

6. Thiriez G, Mougey C, Vermeylen D, et al. Altered autonomic control in preterm newborns with impaired neurological outcomes. Clin Auton Res 2015;25:233-42.

7. Pal GK, Pal P, Nanda N, Amudharaj D, Adithan C. Cardiovascular dysfunctions and sympathovagal imbalance in hypertension and prehypertension: physiological perspectives. Future Cardiol 2013;9:53-69.

8. Horne RS. Cardio-respiratory control during sleep in infancy. Paediatr Respir Rev 2014;15:163-9.

9. Fyfe KL, Yiallourou SR, Wong FY, Horne RS. The development of cardiovascular and cerebral vascular control in preterm infants. Sleep Med Rev 2014;18:299-310.

10. Nijhuis IJ, ten Hof J, Mulder EJ, et al. Fetal heart rate in relation to its variation in normal and growth retarded fetuses. Eur J Obstet Gynecol Reprod Biol 2000;89:27-33.

11. Vinkesteijn AS, Struijk PC, Ursem NT, Hop WC, Wladimiroff JW. Fetal heart rate and umbilical artery flow velocity variability in intrauterine growth restriction: a matched controlled study. Ultrasound Obstet Gynecol 2004;23:461-5.

12. Sriram B, Mencer MA, McKelvey S, et al. Differences in the sleep states of iugr and low-risk fetuses: an mcg study. Early Hum Dev 2013;89:815-9.

13. Signorini MG, Magenes G, Cerutti S, Arduini D. Linear and nonlinear parameters for the analysis of fetal heart rate signal from cardiotocographic recordings. IEEE Trans Biomed Eng 2003;50:365-74.

14. Cohen E, Wong FY, Horne RS, Yiallourou SR. Intrauterine growth restriction: impact on cardiovascular development and function throughout infancy. Pediatr Res 2016;79:821-30.

15. Spassov L, Curzi-Dascalova L, Clairambault J, et al. Heart rate and heart rate variability during sleep in small-for-gestational age newborns. Pediatr Res 1994;35:500-5.

16. Padhye NS, Verklan M, Brazdeikis A, Williams AL, Khattak AZ, Lasky RE. A comparison of fetal and neonatal heart rate variability at similar postmenstrual ages. Conf Proc IEEE Eng Med Biol Soc 2008;2008:2801-4.

17. Golder V, Hepponstall M, Yiallourou SR, Odoi A, Horne RS. Autonomic cardiovascular control in hypotensive critically ill preterm infants is impaired during the first days of life. Early Hum Dev 2013;89:419-23.

18. Brew N, Walker D, Wong FY. Cerebral vascular regulation and brain injury in preterm infants. Am J Physiol Regul Integr Comp Physiol 2014;306:R773-86.

19. Cohen E, Baerts W, van Bel F. Brain-sparing in intrauterine growth restriction: considerations for the neonatologist. Neonatology 2015;108: $269-76$.
20. Cohen E, Dix L, Baerts W, Alderliesten T, Lemmers P, van Bel F. Reduction in cerebral oxygenation due to patent ductus arteriosus is pronounced in small-for-gestational-age neonates. Neonatology 2016;111:126-32.

21. Miller SL, Huppi PS, Mallard C. The consequences of fetal growth restriction on brain structure and neurodevelopmental outcome. J Physiol 2016;594:807-23.

22. Doussard-Roosevelt JA, McClenny BD, Porges SW. Neonatal cardiac vagal tone and school-age developmental outcome in very low birth weight infants. Dev Psychobiol 2001;38:56-6.

23. Goulding RM, Stevenson NJ, Murray DM, Livingstone V, Filan PM, Boylan GB. Heart rate variability in hypoxic ischemic encephalopathy: correlation with eeg grade and 2-y neurodevelopmental outcome. Pediatr Res 2015;77:681-7.

24. Sanz-Cortes M, Egana-Ugrinovic G, Zupan R, Figueras F, Gratacos E. Brainstem and cerebellar differences and their association with neurobehavior in term small-for-gestational-age fetuses assessed by fetal MRI. Am J Obstet Gynecol 2014;210:e451-8.

25. Gasior JS, Sacha J, Jelen PJ, Pawlowski M, Werner B, Dabrowski MJ. Interaction between heart rate variability and heart rate in pediatric population. Front Physiol 2015;6:385.

26. Buchhorn R, Hammersen A, Bartmus D, Bursch J. The pathogenesis of heart failure in infants with congenital heart disease. Cardiol Young 2001:11:498-504.

27. Sajadieh A, Nielsen OW, Rasmussen V, Hein HO, Abedini S, Hansen JF. Increased heart rate and reduced heart-rate variability are associated with subclinical inflammation in middle-aged and elderly subjects with no apparent heart disease. Eur Heart J 2004;25:363-70.

28. Galland BC, Taylor BJ, Bolton DP, Sayers RM. Heart rate variability and cardiac reflexes in small for gestational age infants. J Appl Physiol (1985) 2006;100:933-9.

29. Cohen G, Vella S, Jeffery H, Lagercrantz H, Katz-Salamon M. Cardiovascular stress hyperreactivity in babies of smokers and in babies born preterm. Circulation 2008;118:1848-53.

30. Van Reempts PJ, Wouters A, De Cock W, Van Acker KJ. Stress responses to tilting and odor stimulus in preterm neonates after intrauterine conditions associated with chronic stress. Physiol Behav 1997;61:419-24.

31. Aziz W, Schlindwein FS, Wailoo M, Biala T, Rocha FC. Heart rate variability analysis of normal and growth restricted children. Clin Auton Res 2012;22:91-7.

32. Dalla Pozza RD, Bechtold S, Putzker S, Bonfig W, Netz H, Schwarz HP. Young adults born small for gestational age: Is reduced baroreceptor sensitivity a risk factor for hypertension? Clin Cardiol 2006;29:215-8.

33. Wolfenstetter A, Simonetti GD, Poschl J, Schaefer F, Wuhl E. Altered cardiovascular rhythmicity in children born small for gestational age. Hypertension 2012;60:865-70.

34. Khalil A, Morales-Rosello J, Khan N, et al. Is cerebroplacental ratio a marker of impaired fetal growth velocity and adverse pregnancy outcome? Am J Obstet Gynecol 2017 Feb 8. pii: S0002-9378(17)30182-5. (doi:10.1016/j.ajog.2017.02.005; e-pub ahead of print).

35. Yiallourou SR, Sands SA, Walker AM, Horne RS. Baroreflex sensitivity during sleep in infants: Impact of sleeping position and sleep state. Sleep 2011;34:725-32.

36. Verdurmen KM, Renckens J, van Laar JO, Oei SG. The influence of corticosteroids on fetal heart rate variability: a systematic review of the literature. Obstet Gynecol Surv 2013;68:811-24.

37. Verdurmen KM, Hulsenboom AD, van Laar JO, Oei SG. Effect of tocolytic drugs on fetal heart rate variability: a systematic review. J Matern Fetal Neonatal Med 2016: 1-8.

38. Sheen TC, Lu MH, Lee MY, Chen SR. Nonreassuring fetal heart rate decreases heart rate variability in newborn infants. Ann Noninvasive Electrocardiol 2014;19:273-8.

39. Hadlock FP, Harrist RB, Martinez-Poyer J. In utero analysis of fetal growth: a sonographic weight standard. Radiology 1991;181:129-33.

40. Gomez O, Figueras F, Fernandez S, et al. Reference ranges for uterine artery mean pulsatility index at 11-41 weeks of gestation. Ultrasound Obstet Gynecol 2008;32:128-32.

41. Acharya G, Wilsgaard T, Berntsen GK, Maltau JM, Kiserud T. Reference ranges for serial measurements of umbilical artery doppler 


\section{Articles | cohen et al.}

indices in the second half of pregnancy. Am J Obstet Gynecol 2005;192: 937-44.

42. Mari G, Deter RL. Middle cerebral artery flow velocity waveforms in normal and small-for-gestational-age fetuses. Am J Obstet Gynecol 1992;166:1262-70.

43. Schechtman VL, Harper RM, Kluge KA. Development of heart rate variation over the first 6 months of life in normal infants. Pediatr Res 1989;26:343-6.

44. Curzi-Dascalova L, Mirmiran M, Manual of Methods for Recording and Analyzing Sleep-Wakefulness States in Preterm and Full-Term Infant. Paris: Inserm, 1996.

45. Yiallourou SR, Sands SA, Walker AM, Horne RS. Maturation of heart rate and blood pressure variability during sleep in term-born infants. Sleep 2012;35:177-86.
46. Anders T, Emde R, Parmelee A. A Manual of Standardized Terminology, Techniques and Criteria for Scoring of States of Sleep and Wakefulness in Newborn Infants. Los Angeles: UCLA Brain Information Service, 1971: 41.

47. de Beer NA, Andriessen P, Berendsen RC, Oei SG, Wijn PF, Oetomo SB. Customized spectral band analysis compared with conventional fourier analysis of heart rate variability in neonates. Physiol Meas 2004;25: 1385-95.

48. Andriessen P, Oetomo SB, Peters C, Vermeulen B, Wijn PF, Blanco CE. Baroreceptor reflex sensitivity in human neonates: the effect of postmenstrual age. J Physiol 2005;568:333-41.

49. Yiallourou SR, Sands SA, Walker AM, Horne RS. Postnatal development of baroreflex sensitivity in infancy. J Physiol 2010;588:2193-03. 